\title{
Rotation-differential rotation relationships for late-type single and bi- nary stars from Doppler imaging
}

\author{
Zs. Kővári ${ }^{1, \star}$, K. Oláh ${ }^{1}$, L. Kriskovics ${ }^{1}$, K. Vida ${ }^{1}$, E. Forgács-Dajka ${ }^{2}$, and K. G. Strassmeier ${ }^{3}$ \\ 1 Konkoly Observatory, Research Center for Astronomy and Earth Sciences, Budapest, Hungary \\ 2 Eötvös University, Department of Astronomy, Budapest, Hungary \\ ${ }^{3}$ Leibniz-Institute for Astrophysics Potsdam (AIP), Germany
}

Received XXXX, accepted XXXX

Published online XXXX

Key words stars: activity-stars: imaging-stars: late-type-starspots-differential rotation

From our sample of spotted late-type stars showing surface differential rotation we find that the relationship between the rotation period and the surface shear coefficient $\alpha=\Delta \Omega / \Omega_{\mathrm{eq}}$ is significantly different for single stars compared to members in close binaries. Single stars follow a general trend that $\alpha$ increases with the rotation period. However, differential rotation of stars in close binary systems shows much weaker dependence on the rotation, if any, suggesting that in such systems tidal forces operate as a controlling mechanism of differential rotation.

Copyright line will be provided by the publisher

\section{Introduction}

Stellar dynamos working in late-type stars generate strong magnetic fields, which, at the end, are manifested in activity phenomena such like starspots. Differential rotation of spotted stars with convective envelopes is of utmost importance in understanding how the dynamo mechanism (re)generates large scale toroidal fields by the so-called $\Omega$-effect. However, magnetic dynamos work diversely in different types of stars.

We have learned that rotation is the main driving force that can sustain the dynamo, still, it is not known what kind of relationship exists between rotation and differential rotation, if any. In principle, with increasing rotation rate the role of differential rotation is getting less significant, i.e., according to the mean field theory, a transition may be hypothesized from $\alpha \Omega$ and $\alpha^{2} \Omega$-type dynamos to $\alpha^{2}$ dynamos (Brun et al. 2015). While in rapidly rotating young latetype $(\mathrm{G}-\mathrm{K})$ stars supposedly $\alpha^{2} \Omega$-type dynamos work, in fully convective low-mass stars or brown dwarfs a pure $\alpha^{2}$ type dynamo may operate, wherein the helical turbulence ( $\alpha$-effect) is dominant and the $\Omega$-effect is basically negligible (Chabrier \& Küker 2006). For the effect of the Rossby number on the dynamo in fully convective $\mathrm{M}$-dwarfs and the presumed transition from $\alpha^{2}$ dynamos to $\alpha \Omega$-type see Yadav et al. (2016).

In RS CVn-type binary systems tidal coupling is responsible for maintaining fast rotation. Also, the gravitational influence of a close companion yields different physical conditions inside a differentially rotating convective bulk, which may imply that dynamos work differently in single

^ Corresponding author: kovari@konkoly.hu stars compared to components of RS CVn systems (Kővári et al. 2012a). Although, the related background theory is still too complex in its predictive power, for now the observational database is wide enough to study the relationship between the rotation rate and the differential rotation.

Starspots are proved to be useful tracers for measuring surface differential rotation on either single stars or members in binary systems (see, e.g., Donati \& Collier Cameron 1997: Kôvári et al.|2012b; Weber 2007; and see also the review by Strassmeier 2009). In this paper we aim to analyze how the differential surface shear depends on the rotation, also, how the relationship is affected by the binarity. We collect surface shear coefficients from the literature, based on the most reliable Doppler imaging and Zeeman-Doppler imaging studies from the past two decades. Observations have already proved the existence of antisolar-type differential rotation (see, e.g., Kôvári \& Oláh 2014, and their references), i.e., when the rotation is the slowest at the equator and increases with latitude towards the pole. In Sect. 5 we suggest a possible scenario which may yield such a peculiar surface rotation pattern on single K-giants arriving at the red giant branch (RGB).

\section{Measuring surface differential rotation}

Stellar surface differential rotation laws are generally written in a quadratic form of

$\Omega(\beta)=\Omega_{\mathrm{eq}}\left(1-\alpha \sin ^{2} \beta\right)$,

where $\Omega(\beta)$ is the angular velocity at $\beta$ latitude, $\Omega_{\mathrm{eq}}$ is the angular velocity at the equator, while the dimensionless relative surface shear $\alpha$ is expressed as $\alpha=\Delta \Omega / \Omega_{\mathrm{eq}}$, where $\Delta \Omega=\Omega_{\mathrm{eq}}-\Omega_{\mathrm{pol}}$ is the absolute shear. In this context $\alpha>0$ 
stands for solar-type differential rotation, when the angular velocity is maximum at the equator, while $\alpha<0$ means antisolar differential rotation, when the equatorial belt rotates the most slowly.

When measuring surface differential rotation of latetype stars, basically two methods are considered, namely the cross-correlation of subsequent Doppler (or ZeemanDoppler) images and the sheared image method, also known as parametric imaging. For the cross-correlation technique two subsequent image reconstructions are needed and the rotationward cross-correlation function map obtained from the images may reveal the differential rotation pattern. However, this pattern can easily be blurred by rapid surface evolution (e.g., emerging a new spot, merging and dissolving spots). On the other hand, when having more than two Doppler images in time series, such unwanted effects can be reduced by averaging all the available cross-correlation maps, yielding a more reliable result. For the detailed description of this average cross-correlation method see, e.g. Kôvári et al. (2015) and the references therein. The sheared image method (e.g. Donati et al. 2000, Petit et al. 2002), however, works even when having only one single Doppler image. The surface shear is incorporated in the reconstruction process as a predefined parameter while image reconstructions are carried out for a meaningful range of the surface shear-equatorial rotation parameter plane. Each Doppler reconstruction has a goodness-of-fit value which may help to find the most probable surface rotation law. We note that this parametric imaging works for less data compared to the cross-correlation technique, but, maybe at the cost of reliability; for critical remarks on this issue see, e.g., Kôvári \& Oláh (2014) and their references. In addition, Kôvári et al. (2014a) demonstrated that a large polar cap, frequently detected in rapid rotators, could also yield false measure of the surface shear when applying parametric imaging.

\section{The collected observational sample}

In this paper we focus on spotted late-type stars, i.e., solartype or later classifications (G-K-M), confining to the evolutionary phase from zero age main sequence up to the RGB. In this evolutionary phase, dynamos in single stars are thought to be influenced mostly by the rotation and spectral type through the related convection zone depth. The dynamo process, however, is expected to be perturbed by tidal effects when having a close companion star. Therefore, when setting up our target list we focus on the single-binary distinction as well.

Our observational sample is based on Doppler imaging and Zeeman-Doppler imaging studies from the literature. Differential rotation measurements from Stokes V, i.e., from tracing magnetic features, usually yield higher shear values than the Stokes I results of the same target (cf., e.g., Petit et al. 2004b, their Table 7; Waite et al.2011, their Fig. 11). This is partially explained by the different anchoring depths of surface spots and magnetic tracers, however, the given errors of the corresponding shear values are higher as well, which suggests more uncertainty for the Stokes V results compared to Stokes I. Therefore, when both available, we preferred choosing Stokes I measurements, which are more compatible with the results from traditional Doppler imaging. In this study, however, we neglect rapidly rotating premain sequence stars, for which differential rotation values often show unexpected irregularities (e.g. Marsden et al. 2011; Waite et al. 2011) possibly due to the vivid evolutionary phase or other unidentified effects. Although, seasonal changes of the rotation period derived from long-term photometric datasets or the spread in the rotation frequency could also be considered as clues for the differential rotation (e.g. Balona \& Abedigamba 2016, Vida et al. 2014), however, photometric analysis enables only a rough estimation of $\alpha$ (without sign), therefore we do not include those results in our list.

Table 1 lists the selected targets together with their basic properties such as $P_{\text {rot }}, T_{\text {eff }}$, luminosity class and binarity along with the measured absolute and relative surface shear values, i.e., $\Delta \Omega$ and $\alpha=\Delta \Omega / \Omega_{\mathrm{eq}}$, respectively, and the corresponding references. The list consists of 37 differential rotation measurements from 24 stars, including 8 single dwarfs, 6 single or effectively single giants, and 5 subgiant and 5 giant members in close binary systems. In the sample 26 detections reflect solar-type differential rotation, while 11 detections are of antisolar type. This latter was found mainly for giants with relatively long rotational periods, being either single stars (e.g., DI Psc, V1192 Ori) or members in RS CVn systems (e.g., $\sigma$ Gem, HKLac). For some targets the differential rotation was determined independently either by different authors, different methods, and/or for different epochs. Usually, these multiple determinations are in good agreement, but there are also divergent or even contradictory results. For instance, a quite peculiar result was reported for IM Peg by Marsden et al. (2007), where the authors used a 2.7 year-long observational dataset to extract 22 differential rotation measurements. It was found, that the $\Delta \Omega$ surface shear had fluctuated between about $-0.7^{\circ} / \mathrm{d}$ and $+2.1 \%$, i.e., between antisolar and solar-type, which is quite dubious. Unsurprisingly, among other possible explanations, the authors considered that the error bars of their method was underestimated, i.e., the scatter of their detections reflected rather an ultimate error instead of real fluctuations. Therefore, in this special case we use their grand average shear value. In this context we note, that in most cases the error bars of the $\alpha$ values given in Table 1 are significantly underestimated and the more realistic relative errors should be around 30-40\% (Kővári et al. 2015, Kővári \& Weber 2004).

\section{Results}

In Fig. 1 we plot the $\alpha=\Delta \Omega / \Omega_{\mathrm{eq}}$ dimensionless surface shear coefficient as a function of $P_{\text {rot }}$. We use absolute val- 
Table 1 Surface differential rotation parameters from Doppler imaging studies.

\begin{tabular}{|c|c|c|c|c|c|c|c|c|}
\hline Star & $P_{\text {rot }}[\mathrm{d}]$ & $\Omega_{\mathrm{eq}}[\% / \mathrm{d}]$ & $\Delta \Omega[\% / \mathrm{d}]$ & $\alpha=\Delta \Omega / \Omega_{\mathrm{eq}}$ & $T_{\text {eff }}[\mathrm{K}]$ & type $^{a}$ & method $^{b}$ & reference $^{c}$ \\
\hline BO Mic & 0.38 & $947.44 \pm 0.034$ & $1.891 \pm 0.172$ & $0.0020 \pm 0.0002$ & 4890 & $\mathrm{~V}, \mathrm{~s}$ & $\operatorname{sim}$ & B05 \\
\hline LOPeg & 0.42 & $851.415 \pm 0.155$ & $2.005 \pm 0.401$ & $0.0024 \pm 0.0005$ & 4600 & $\mathrm{~V}, \mathrm{~s}$ & $\operatorname{sim}$ & BCL05 \\
\hline HK Aqr & 0.43 & $834.97 \pm 0.132$ & $0.2808 \pm 0.115$ & $0.0003 \pm 0.0001$ & 3700 & $\mathrm{~V}, \mathrm{~s}$ & $\operatorname{sim}$ & BJC04 \\
\hline AB Dor & 0.51 & $701.459 \pm 0.014$ & $3.2315 \pm 0.701$ & $0.0046 \pm 0.001$ & 5000 & $\mathrm{~V}, \mathrm{~s}$ & $\operatorname{ccf}$ & DC97 \\
\hline V557 Car & 0.57 & $638.218 \pm 0.458$ & $1.43 \pm 0.860$ & $0.0022 \pm 0.001$ & 5800 & $\mathrm{~V}, \mathrm{~s}$ & $\operatorname{sim}$ & MWC05 \\
\hline V557 Car & 0.57 & $641.713 \pm 0.573$ & $8.02 \pm 0.573$ & $0.0123 \pm 0.0008$ & 5800 & $\mathrm{~V}, \mathrm{~s}$ & $\operatorname{sim}$ & MWC05 \\
\hline LQ Hya & 1.60 & $224.9 \pm 0.02$ & $0.573 \pm 0.172$ & $0.0025 \pm 0.001$ & 5019 & $\mathrm{~V}, \mathrm{~s}$ & $\operatorname{sim}$ & DCP03 \\
\hline LQ Hya & 1.60 & $224.9 \pm 0.02$ & $11.12 \pm 1.15$ & $0.0494 \pm 0.005$ & 5019 & $\mathrm{~V}, \mathrm{~s}$ & $\operatorname{sim}$ & DCP03 \\
\hline LQ Hya & 1.60 & $225.287 \pm 4.01$ & $1.261 \pm 0.458$ & $0.0056 \pm 0.0022$ & 5070 & $\mathrm{~V}, \mathrm{~s}$ & $\operatorname{ccf}$ & KSG04 \\
\hline EIEri & 1.95 & $188.0 \pm 0.2$ & $7.0 \pm 1.9$ & $0.036 \pm 0.01$ & 5500 & $\mathrm{IV}, \mathrm{b}$ & $\operatorname{ccf}$ & KWF09 \\
\hline FK Com & 2.40 & 149.769 & $0.015 \pm 0.030$ & $0.0001 \pm 0.0002$ & 5000 & III,s & $\operatorname{ccf}$ & KBHOO \\
\hline V711 Tau & 2.84 & $127.305 \pm 0.275$ & $-0.083 \pm 0.467$ & $-0.001 \pm 0.004$ & 4750 & $\mathrm{IV}, \mathrm{b}$ & $\operatorname{sim}$ & DCP03 \\
\hline V711 Tau & 2.84 & $127.323 \pm 0.241$ & $1.215 \pm 0.464$ & $0.010 \pm 0.004$ & 4750 & $\mathrm{IV}, \mathrm{b}$ & $\operatorname{sim}$ & DCP03 \\
\hline V711 Tau & 2.84 & $127.432 \pm 0.023$ & $0.871 \pm 0.047$ & $0.007 \pm 0.001$ & 4750 & $\mathrm{IV}, \mathrm{b}$ & $\operatorname{sim}$ & PDV04 \\
\hline V1794 Cyg & 3.30 & $110.63 \pm 0.974$ & $4.927 \pm 1.202$ & $0.045 \pm 0.011$ & 5350 & III,s & $\operatorname{sim}$ & PDO04 \\
\hline V1794 Cyg & 3.30 & $110.69 \pm 1.891$ & $4.526 \pm 3.495$ & $0.041 \pm 0.032$ & 5350 & III,s & $\operatorname{sim}$ & PDO04 \\
\hline V1794 Cyg & 3.30 & $110.81 \pm 1.031$ & $3.782 \pm 1.604$ & $0.034 \pm 0.011$ & 5350 & III,s & $\operatorname{sim}$ & PDO04 \\
\hline UZLib & 4.77 & 75.50 & $-2.039 \pm 0.227$ & $-0.027 \pm 0.003$ & 4800 & $\mathrm{IV}, \mathrm{b}$ & $\operatorname{ccf}$ & VKS07 \\
\hline HU Vir & 10.39 & $34.38 \pm 0.15$ & $-1.01 \pm 0.23$ & $-0.029 \pm 0.005$ & 4700 & $\mathrm{IV}, \mathrm{b}$ & $\operatorname{ccf}$ & HSK16 \\
\hline IL Hya & 12.73 & 28.02 & $0.84 \pm 0.34$ & $0.030 \pm 0.012$ & 4500 & $\mathrm{IV}, \mathrm{b}$ & $\operatorname{sim}$ & KW04 \\
\hline IL Hya & 12.73 & 28.44 & $0.76 \pm 0.28$ & $0.027 \pm 0.010$ & 4500 & $\mathrm{IV}, \mathrm{b}$ & $\operatorname{ccf}$ & KW04 \\
\hline IL Hya & 12.73 & $28.28 \pm 0.03$ & $1.43 \pm 0.15$ & $0.050 \pm 0.010$ & 4500 & $\mathrm{IV}, \mathrm{b}$ & $\operatorname{ccf}$ & KKO14 \\
\hline DPCVn & 14.01 & $25.336 \pm 0.044$ & $-0.875 \pm 0.411$ & $-0.035 \pm 0.016$ & 4600 & III,s & $\operatorname{ccf}$ & KKS13 \\
\hline$\zeta$ And & 17.77 & $19.02 \pm 0.16$ & $0.95 \pm 0.07$ & $0.050 \pm 0.004$ & 4600 & III,b & $\operatorname{ccf}$ & KBS07 \\
\hline$\zeta$ And & 17.77 & $20.689 \pm 0.055$ & $1.138 \pm 0.045$ & $0.055 \pm 0.0022$ & 4600 & III,b & $\operatorname{ccf}$ & KKK12 \\
\hline DI Psc & 18.07 & $19.7 \pm 0.15$ & $-1.63 \pm 0.41$ & $-0.083 \pm 0.021$ & 4600 & III,s & $\operatorname{ccf}$ & KKV14 \\
\hline$\sigma \mathrm{Gem}$ & 19.60 & $18.26 \pm 0.07$ & $-0.840 \pm 0.13$ & $-0.046 \pm 0.01$ & 4600 & III,b & $\operatorname{ccf}$ & KKK15 \\
\hline V2075 Cyg & 22.09 & $19.297 \pm 0.074$ & $-0.772 \pm 0.386$ & $-0.040 \pm 0.02$ & 4700 & III,b & $\operatorname{sim}$ & WSW05 \\
\hline V2075 Cyg & 22.62 & $16.043 \pm 0.229$ & $0.241 \pm 0.048$ & $0.015 \pm 0.003$ & 4600 & III,b & $\operatorname{ccf}$ & ÖCK16 \\
\hline KU Peg & 23.90 & $15.5138 \pm 0.0735$ & $0.6215 \pm 0.092$ & $0.040 \pm 0.006$ & 4440 & III,s & $\operatorname{ccf}$ & KKS16 \\
\hline HK Lac & 24.20 & $14.876 \pm 0.062$ & $-0.744 \pm 0.744$ & $-0.05 \pm 0.05$ & 4700 & III,b & $\operatorname{sim}$ & WSW05 \\
\hline IM Peg & 24.25 & $14.845 \pm 0.15$ & $-0.742 \pm 0.742$ & $-0.05 \pm 0.05$ & 4500 & III,b & $\operatorname{sim}$ & WSW05 \\
\hline IM Peg & 24.65 & $14.931 \pm 0.011$ & $0.814 \pm 0.040$ & $0.054 \pm 0.003$ & 4500 & III,b & $\operatorname{sim}$ & MBD07 \\
\hline V1192 Ori & 25.30 & $13.87 \pm 0.22$ & $-1.73 \pm 0.67$ & $-0.125 \pm 0.050$ & 4500 & III,s & $\operatorname{ccf}$ & SKW03 \\
\hline V1192 Ori & 28.30 & $12.695 \pm 0.034$ & $-1.414 \pm 0.148$ & $-0.11 \pm 0.02$ & 4305 & III,s & $\operatorname{ccf}$ & KSO17 \\
\hline Sun & 25.38 & 14.37 & 2.86 & 0.20 & 5780 & $\mathrm{~V}, \mathrm{~s}$ & str & BVW86 \\
\hline 61 Cyg A & 34.20 & $10.31 \pm 0.286$ & $2.292 \pm 0.573$ & $0.22 \pm 0.056$ & 4545 & $\mathrm{~V}, \mathrm{~s}$ & $\operatorname{sim}$ & BJM16 \\
\hline
\end{tabular}

${ }^{a} \mathrm{~V}$ : dwarf, IV: subgiant, III: giant, s: single or effectively single, b: member of a close binary system

${ }^{b}$ sim: sheared image method, ccf: cross-correlation method, str: spot tracking technique

${ }^{c}$ B05: Barnes (2005), BCL05: Barnes et al. (2005b), BJC04: Barnes et al. (2004), DC97: Donati \& Collier Cameron (1997), MWC05: Marsden et al. (2005), DCP03: Donati et al. (2003), PDV04 Petit et al. (2004b), KSG04: Kővári et al. (2004), KWF09: Kővári et al. (2009), KBH00: Korhonen et al. (2000), PDO04: Petit et al. (2004a), VKS07: Vida et al. (2007), HSK16: Harutyunyan et al. (2016), KW04: Kővári \& Weber(2004), KKO14: Kővári et al.(2014b), KKS13: Kővári et al. (2013), KBS07: Kővári et al. (2007), KKK12: Kővári et al. (2012b), KKV14: Kriskovics et al. (2014), KKK15: Kővári et al. (2015), WSW05: Weber et al. (2005), ÖCK16: Özdarcan et al. (2016), KKS16: Kővári et al. (2016), MBD07: Marsden et al. (2007), SKW03: Strassmeier et al. (2003), KSO17: Kővári et al. (2017), BVW86: Balthasar et al.(1986), BJM16: Boro Saikia et al. (2016)

ues of $\alpha$ because in this context there is no difference of substance between solar-type and antisolar differential rotation, since the field amplification due to the $\Omega$-effect is related directly to the shear, not to its direction. The overall distribution of the datapoints reflects a trend that the longer the rotation period, the higher the surface shear coefficient (cf. Kôvári \& Oláh 2014). On the other hand, there is a significant difference between the distribution of the open symbols representing single stars and the grey-filled symbols representing stars in close binary systems. The two types of symbols are fitted (unweighted) by two different linear functions. The basic difference between the fits suggests, that the surface shear coefficient, i.e., the differential rotation is somehow confined in binary systems. The shear coefficient for close binary members grows only slowly towards the longer periods, and does not reach as high values as found for slowly rotating single stars. The fit for single stars (dotted line in Fig. 11 yields $|\alpha| \propto(0.0049 \pm 0.0001) P_{\text {rot }}[\mathrm{d}]$ while 


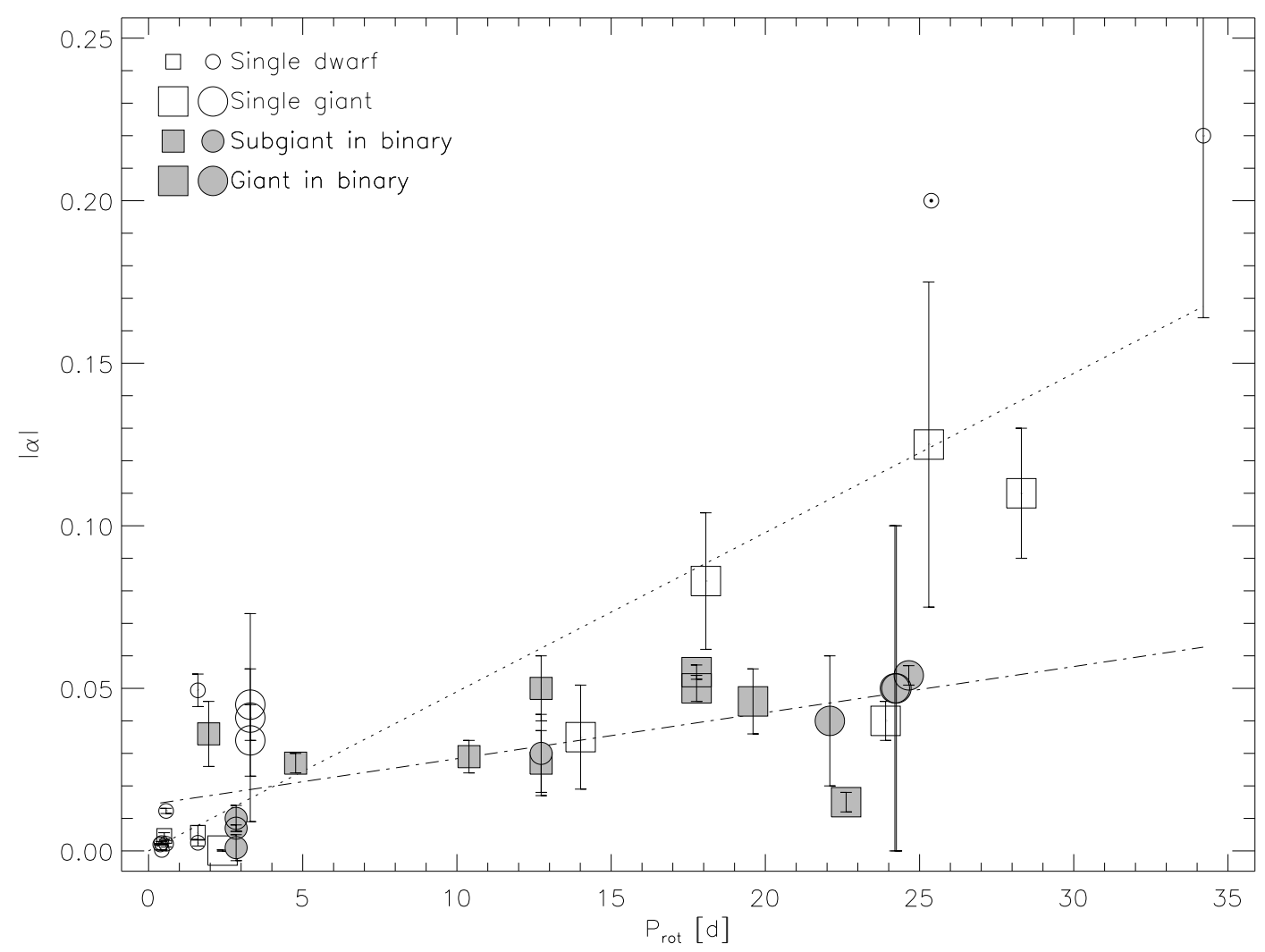

Fig. 1 The absolute values of the dimensionless surface shear parameter $\alpha$ from Table 1 vs. rotation period. Open symbols are single stars, grey-filled symbols are members of close binary systems. Circles represent results by applying the sheared image method while rectangles are results obtained from cross-correlation technique. Dotted line represents a linear fit to the single stars while dash-dotted line fits the binary members of our sample.

the fit for the binary members (dash-dotted line) has a restrained slope of $0.0014 \pm 0.0003$. Finally we note, that plotting $|\alpha|$ as a function of $P_{\text {rot }}$ is essential, since a plot similar to Barnes et al. (2005a, Fig. 3), where $\log _{10} \Delta \Omega$ was plotted as a function of $\log _{10} \Omega_{\mathrm{eq}}$, would not reveal such striking difference between single stars and binary members. In the next section we further discuss on this exciting result.

In Fig. 2] we plotted $\Delta \Omega$ absolute surface shear as a function of the effective temperature. The best fit for the unweighted data points is in agreement with the early result by Barnes et al. (2005a their Fig. 2), suggesting an overall trend that $\Delta \Omega$ is growing with the temperature. Assuming a power-law in the form of $\Delta \Omega \propto T_{\text {eff }}^{p}$, we get $p=5.8 \pm 1.0$ for our sample. In the end we mention, that single stars and binaries, i.e., open and filled symbols, respectively, are not particularly separated in Fig. 2 .

\section{Discussion}

\subsection{Differential rotation of single $G-K-M$ stars}

Single late-type stars seem to follow a simple trend that fast-rotators have small relative shear, while slower rotators generally perform larger relative surface shear (with a large scatter, though). Considering the true error bars which are very likely larger than the estimated values in Table 1 (cf. Sect. 3), the dotted line nicely fits the observations, except one measurement of the fast-rotating LQ Hya at $P_{\text {rot }}=1.6 \mathrm{~d}$. There are three detections listed in Table 1 for this target, two independent results among them are in agreement, claiming a weak differential rotation with $\alpha=0.0025$ and 0.0056 (Donati et al. 2003; Kővári et al. 2004, respectively), while the third result, $\alpha=0.0494$ (also from Donati et al. 2003 ) is higher by an order of a magnitude compared to the other two. This fluctuation, real or not, can be associated with the changes in the kinetic energy, which was estimated to be 10 per cent of the total stellar luminosity of LQHya (Donati et al.2003). However, this explanation is not applicable on a time scale of a year or so. It is more likely that, similarly to the case of IM Peg (Marsden et al.2007) already mentioned in Sect. 3, the fluctuating detections by Donati et al. (2003) reflect uncovered inner errors of the sheared image method applied (for more discussion see Sect. 3.3.1 last paragraph in Kővári \& Oláh 2014). Therefore, we consider this higher shear as an erroneous detection and the smaller values as more realistic. However, despite the discrepancy, we did not to exclude this datapoint from the fits shown in Fig. 1 and Fig. 2 . 


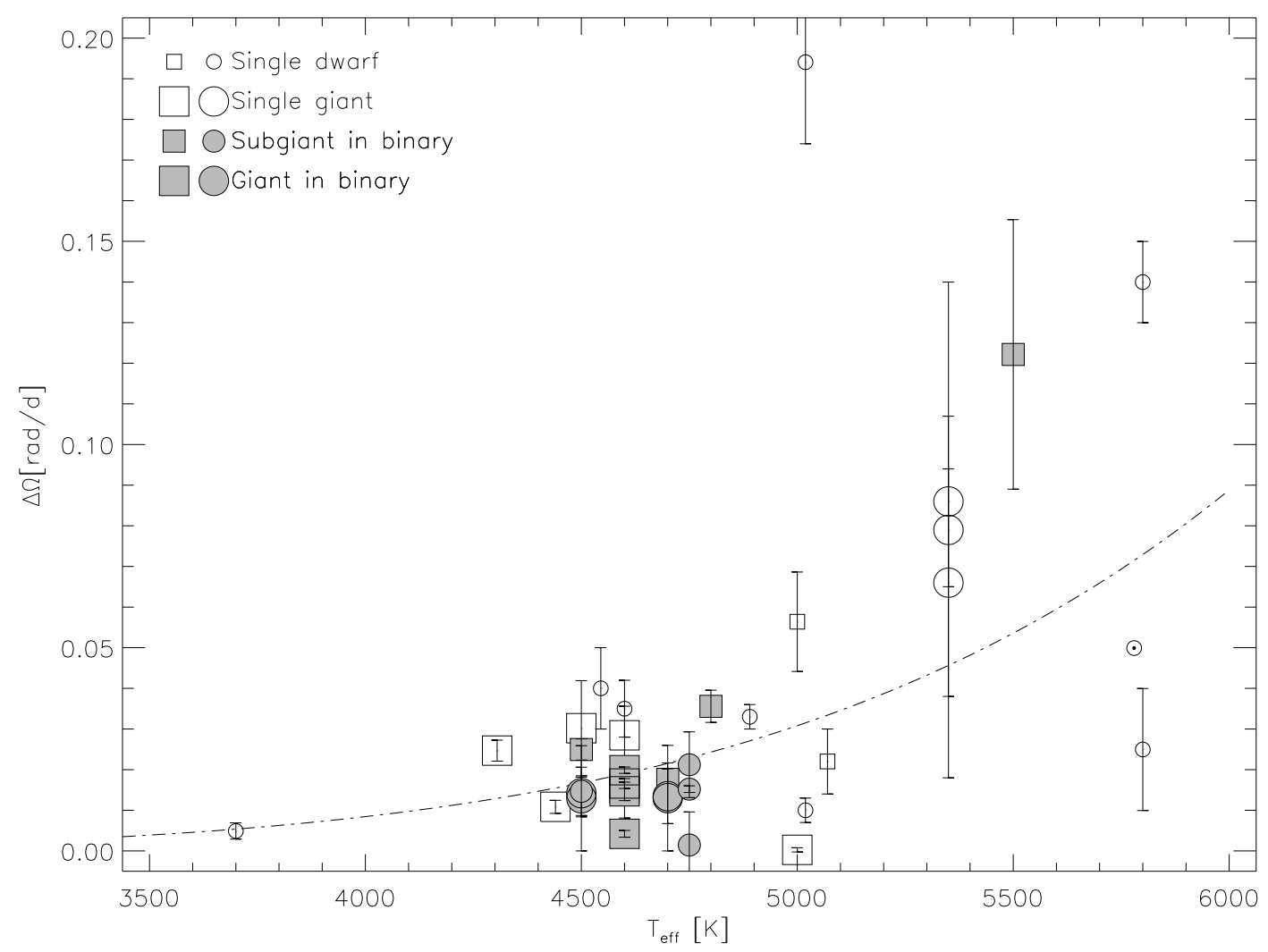

Fig. 2 Absolute surface shear vs. effective temperature. Dash-dotted line represents an unweighted power-law fit to all datapoints. Otherwise as in Fig. 1 .

Smaller relative shear is preferred also by theory in fastrotating dwarf stars. A strong shear would be reduced by the so-called $\Omega$-quenching, because an intensified $\Omega$-effect would yield more and more dominant toroidal fields, and the related magnetic energy would increase until the counteraction of the Lorentz-force on the plasma flow. Consequently, the differential rotation would reduce dramatically (see, e.g., Brun et al. 2015, and their references). According to Forgács-Dajka (2004), a similar mechanism prevents the differential rotation from penetrating down into the solar radiative interior. Note, that the differential rotation is highly quenched in fully convective M-stars as well, until the rotation is fast enough (Yadav et al. 2016). The model by Kitchatinov \& Rüdiger (1999) suggests that $\Delta \Omega / \Omega \sim \Omega^{-n}$, where $n \approx 1$ for G-K dwarfs, i.e., $\Delta \Omega / \Omega \propto P_{\text {rot }}$. The direct relationship between the dimensionless surface shear parameter and the equatorial period gives $\alpha \approx P_{\text {eq }}[\mathrm{d}] / 100$ (see also Küker et al.2011), which agrees with the preferred smaller shear values of our fast-rotating single dwarfs. The (unweighted) linear fit for the collected sample of single and effectively single stars in Fig. 1 suggests $\alpha \approx P_{\text {eq }}[\mathrm{d}] / 200$, i.e., a two times larger denominator, still the same order. Finally, this dependence is supported also by Kepler photometry (Balona \& Abedigamba 2016) who found $\Delta \Omega / \Omega \propto \Omega^{-0.8}$ for G-stars and $\Delta \Omega / \Omega \propto \Omega^{-1.1}$ for K-stars, separately, i.e., with exponents close to -1 , as suggested thereinbefore.

\subsection{Antisolar differential rotation of Li-rich single giants}

In our sample there are three single K-giants, namely DPCVn, DIPsc, and V1192 Ori, all three located at the RGB, rotate rapidly, and show enhanced surface Li abundance (Kővári et al. 2013; Kriskovics et al. 2014, Strassmeier et al. 2003). Moreover, these are the only known single giants that perform antisolar type surface differential rotation (cf. Table 1). The enriched surface lithium at the RGB together with antisolar differential rotation may imply a common origin of these properties, as suggested first by Kriskovics et al. (2014), but see also Kővári et al. (2017). The relatively rapid rotation of these objects can be explained by the interaction between the deepening convective envelope and the fast rotating core (see, e.g. Charbonnel \& Lagarde 2010, Simon \& Drake 1989). The first dredgeup episode on the RGB is responsible for the dilution of the surface lithium, however these three giants show enriched surface lithium. This controversy may be resolved by the so-called cool-bottom processes (Sackmann \& Boothroyd 1992, Wasserburg et al. (1995) where cool material from the bottom of the convective envelope is brought down to hotter layers and exposed to partial $\mathrm{H}$ burning to produce lithium and other light elements. Then, the lithium-rich material is transported back to the convective envelope by some deep 
circulation, wherefrom the lithium can reach the surface by convective mixing and/or meridional circulation.

On the other hand, strong mixing by turbulent convection can equilibrate angular momentum, inducing antisolar differential rotation (Aurnou et al. 2007). Therefore we believe that, in case of DPCVn, DI Psc, and V1192 Ori, antisolar differential rotation and surface lithium enrichment are indeed related attributes. In a possible scenario deep mixing is supposed to bring up fresh lithium along with angular momentum from the hotter layers into the convective envelope, and from there meridional circulation and/or turbulent convection may transport lithium as well as angular momentum towards the surface. However, confirming this scenario needs further investigation of antisolar type differential rotation among single Li-rich giants at the RGB.

\subsection{The gravitational effect of a close companion in close binary systems}

Tidal coupling in RS CVn systems is responsible for maintaining fast rotation, and so makes magnetic dynamo work in the late-type companions. Moreover, tidal effects may organize preferred longitudes, as well as latitudes of activity (Oláh 2007). Aspherical distortion of the active component in an RS CVn-type binary caused by a close companion can explain the emergence of magnetic flux at preferred longitudes locked to the orbital frame, therefore the degree of the deformation could also account for disparate rotation laws (Kôvári et al.|2012a). Theoretical calculations by Scharlemann (1982) demonstrated how physical parameters of such a close binary determine the developing corotation latitude, where rotation is locked to the orbital revolution. Tidal forces may play an important role also in inducing meridional circulation, therefore antisolar differential rotation (Kitchatinov \& Rüdiger 2004). In any way soever, tidal forces do interact with the differentially rotating convective bulk and produce a distinct difference between the relative surface shear measured on binary components compared to single stars of similar type. Indeed, according to Fig. 1 tidal forces seem to confine differential rotation, i.e., $|\alpha|$ may slightly be enhanced for fast-rotating binaries $\left(P_{\text {rot }} \lesssim 2-3\right.$ d) compared to single stars, but the relative shear is firmly suppressed for slower rotating $\left(P_{\text {rot }} \gtrsim 10 \mathrm{~d}\right)$ binary members.

\subsection{The effective temperature-absolute shear dependency}

Theoretical predictions agree that the surface shear is strongly dependent on spectral type (e.g., Kitchatinov \& Rüdiger 1999, Küker \& Rüdiger 2005, 2011). According to our observational result (see Fig. 2) the $\Delta \Omega$ absolute shear slightly increases between $3500-6000 \mathrm{~K}$ towards earlier spectral types. (The only strikingly loose datapoint at the top of the figure is a possible misdetermination for LQHya, cf. Sect. 5.1) Our fitted power-law of $\Delta \Omega \propto T_{\text {eff }}^{p}$ with $p=5.8 \pm 1.0$ suggests still a strong dependency on the temperature, but with a significantly lower exponent compared to $p=8.92 \pm 0.31$ from the early result of Barnes et al. (2005a their Fig. 2). Our result, however, is based on a three times richer and more homogeneous sample of latetype stars. Nevertheless, the lower exponent fits better the theoretical results, which is supported also by Reinhold \& Gizon (2015, see their Fig. 12), where a similar dependency was obtained for G-K spectral types using an extended $\mathrm{Ke}$ pler dataset.

\section{Conclusions}

- We suggest alternative rotation-differential rotation relationships for late-type single stars and binaries. We find an overall trend that the differential rotation represented by the relative surface shear increases with the rotation period, however the dependency is much stronger for single stars. On the other hand, in close binary systems tidal forces confine differential rotation, and therefore, the relative shear is firmly suppressed towards longer periods. We note however, that despite the growing number of individual differential rotation measurements, the available sample is still too small to be representative, and more observations would definitely be essential to improve the statistics.

- So far we know only three single RGB giants DPCVn, DIPsc, and V1192 Ori that perform antisolar surface differential rotation, in addition, all three show surface lithium enrichment. We believe that, in case of these three stars, the peculiar rotation pattern and the lithium enrichment are related attributes, implying a common origin.

- We confirm that the absolute surface shear $\Delta \Omega$ is strongly dependent on the effective temperature. Our unweighted best fit suggests a power-law dependency of $\Delta \Omega \propto T_{\mathrm{eff}}^{5.8 \pm 1.0}$.

Acknowledgements. We thank the anonymous referee for the helpful comments that improved the paper. Authors are grateful to the Hungarian National Research, Development and Innovation Office grants OTKA K-109276 and OTKA K-113117. KV is supported by the Bolyai János Research Scholarship of the Hungarian Academy of Sciences. This paper is partly based on data obtained with the STELLA robotic telescopes in Tenerife, an AIP facility jointly operated by AIP and IAC (https://stella.aip.de/) and by the Amadeus APT jointly operated by AIP and Fairborn Observatory in Arizona. We are grateful to the ministry for research and culture of the State of Brandenburg (MWFK) and the German federal ministry for education and research (BMBF) for their continuous support. The authors acknowledge the support of the German Deutsche Forschungsgemeinschaft, DFG through projects KO2320/1 and STR645/1.

\section{References}

Aurnou, J., Heimpel, M., \& Wicht, J. 2007, Icarus, 190, 110

Balona, L. A. \& Abedigamba, O. P. 2016, MNRAS, 461, 497

Balthasar, H., Vazquez, M., \& Woehl, H. 1986, A\&A, 155, 87 
Barnes, J. R. 2005, MNRAS, 364, 137

Barnes, J. R., Collier Cameron, A., Donati, J.-F., et al. 2005a, MNRAS, 357, L1

Barnes, J. R., Collier Cameron, A., Lister, T. A., Pointer, G. R., \& Still, M. D. 2005b, MNRAS, 356, 1501

Barnes, J. R., James, D. J., \& Collier Cameron, A. 2004, MNRAS, 352,589

Boro Saikia, S., Jeffers, S. V., Morin, J., et al. 2016, A\&A, 594, A29

Brun, A. S., García, R. A., Houdek, G., Nandy, D., \& Pinsonneault, M. 2015, Space Sci. Rev., 196, 303

Chabrier, G. \& Küker, M. 2006, A\&A, 446, 1027

Charbonnel, C. \& Lagarde, N. 2010, A\&A, 522, A10

Donati, J.-F. \& Collier Cameron, A. 1997, MNRAS, 291, 1

Donati, J.-F., Collier Cameron, A., \& Petit, P. 2003, MNRAS, 345, 1187

Donati, J.-F., Mengel, M., Carter, B. D., et al. 2000, MNRAS, 316, 699

Forgács-Dajka, E. 2004, A\&A, 413, 1143

Harutyunyan, G., Strassmeier, K. G., Künstler, A., Carroll, T. A., \& Weber, M. 2016, A\&A, 592, A117

Kővári, Zs., Bartus, J., Kriskovics, L., et al. 2012a, in IAU Symposium, Vol. 282, From Interacting Binaries to Exoplanets: Essential Modeling Tools, ed. M. T. Richards \& I. Hubeny, 197-198

Kővári, Zs., Bartus, J., Kriskovics, L., Vida, K., \& Oláh, K. 2014a, in IAU Symposium, Vol. 302, Magnetic Fields throughout Stellar Evolution, ed. P. Petit, M. Jardine, \& H. C. Spruit, 198-199

Kővári, Zs., Bartus, J., Strassmeier, K. G., et al. 2007, A\&A, 463, 1071

Kővári, Zs., Korhonen, H., Kriskovics, L., et al. 2012b, A\&A, 539, A50

Kővári, Zs., Korhonen, H., Strassmeier, K. G., et al. 2013, A\&A, 551, A2

Kővári, Zs., Kriskovics, L., Künstler, A., et al. 2015, A\&A, 573, A98

Kővári, Zs., Kriskovics, L., Oláh, K., et al. 2014b, in IAU Symposium, Vol. 302, Magnetic Fields throughout Stellar Evolution, ed. P. Petit, M. Jardine, \& H. C. Spruit, 379-380

Kővári, Zs., Künstler, A., Strassmeier, K. G., et al. 2016, A\&A, 596, A53

Kővári, Zs. \& Oláh, K. 2014, Space Sci. Rev., 186, 457

Kővári, Zs., Strassmeier, K. G., Carroll, T. A., et al. 2017, ArXiv e-prints

Kővári, Zs., Strassmeier, K. G., Granzer, T., et al. 2004, A\&A, 417, 1047

Kővári, Zs., Washuettl, A., Foing, B. H., et al. 2009, in American Institute of Physics Conference Series, Vol. 1094, 15th Cambridge Workshop on Cool Stars, Stellar Systems, and the Sun, ed. E. Stempels, 676-679

Kôvári, Zs. \& Weber, M. 2004, Publications of the Astronomy Department of the Eotvos Lorand University, 14, 221

Kitchatinov, L. L. \& Rüdiger, G. 1999, A\&A, 344, 911

Kitchatinov, L. L. \& Rüdiger, G. 2004, Astronomische Nachrichten, 325, 496

Korhonen, H., Berdyugina, S. V., Hackman, T., Strassmeier, K. G., \& Tuominen, I. 2000, A\&A, 360, 1067

Kriskovics, L., Kővári, Zs., Vida, K., Granzer, T., \& Oláh, K. 2014, A\&A, 571, A74

Küker, M. \& Rüdiger, G. 2005, Astronomische Nachrichten, 326, 265

Küker, M. \& Rüdiger, G. 2011, Astronomische Nachrichten, 332,
933

Küker, M., Rüdiger, G., \& Kitchatinov, L. L. 2011, A\&A, 530, A48

Marsden, S. C., Berdyugina, S. V., Donati, J.-F., Eaton, J. A., \& Williamson, M. H. 2007, Astronomische Nachrichten, 328, 1047

Marsden, S. C., Jardine, M. M., Ramírez Vélez, J. C., et al. 2011, MNRAS, 413, 1939

Marsden, S. C., Waite, I. A., Carter, B. D., \& Donati, J.-F. 2005, MNRAS, 359, 711

Oláh, K. 2007, in IAU Symposium, Vol. 240, Binary Stars as Critical Tools \& Tests in Contemporary Astrophysics, ed. W. I. Hartkopf, P. Harmanec, \& E. F. Guinan, 442-452

Özdarcan, O., Carroll, T. A., Künstler, A., et al. 2016, A\&A, 593, A123

Petit, P., Donati, J.-F., \& Collier Cameron, A. 2002, MNRAS, 334, 374

Petit, P., Donati, J.-F., Oliveira, J. M., et al. 2004a, MNRAS, 351, 826

Petit, P., Donati, J.-F., Wade, G. A., et al. 2004b, MNRAS, 348, 1175

Reinhold, T. \& Gizon, L. 2015, A\&A, 583, A65

Sackmann, I.-J. \& Boothroyd, A. I. 1992, ApJ, 392, L71

Scharlemann, E. T. 1982, ApJ, 253, 298

Simon, T. \& Drake, S. A. 1989, ApJ, 346, 303

Strassmeier, K. G. 2009, A\&A Rev., 17, 251

Strassmeier, K. G., Kratzwald, L., \& Weber, M. 2003, A\&A, 408, 1103

Vida, K., Kővári, Zs., Švanda, M., et al. 2007, Astronomische Nachrichten, 328, 1078

Vida, K., Oláh, K., \& Szabó, R. 2014, MNRAS, 441, 2744

Waite, I. A., Marsden, S. C., Carter, B. D., et al. 2011, MNRAS, 413, 1949

Wasserburg, G. J., Boothroyd, A. I., \& Sackmann, I.-J. 1995, ApJ, 447, L37

Weber, M. 2007, Astronomische Nachrichten, 328, 1075

Weber, M., Strassmeier, K. G., \& Washuettl, A. 2005, Astronomische Nachrichten, 326, 287

Yadav, R. K., Christensen, U. R., Wolk, S. J., \& Poppenhaeger, K. 2016, ApJ, 833, L28 